\title{
A Simulator for Testing Planar Upper Extremity Rehabilitation Robot Control Algorithms
}

\author{
K. P. Ayodele ${ }^{\mathrm{a}, *}$, O. T. Akinniyi ${ }^{\mathrm{a}}$, A. O. Oluwatope ${ }^{\mathrm{b}}$, A. M. Jubril ${ }^{\mathrm{a}}$, A. O. \\ Ogundele $^{c}$, M. A. Komolafe ${ }^{\mathrm{d}}$ \\ ${ }^{a}$ Department of Electronic and Electrical Engineering, Obafemi Awolowo University, Ile-Ife, Osun State, \\ NIGERIA. \\ ${ }^{b}$ Department of Computer Science and Engineering, Obafemi Awolowo University, Ile-Ife, Osun State, NIGERIA. \\ ${ }^{c}$ Department of Medical Rehabilitation, Obafemi Awolowo University Teaching Hospital, Ile-Ife, Osun State, \\ NIGERIA. \\ ${ }^{d}$ Department of Computer Science and Engineering, Obafemi Awolowo University, Ile-Ife, Osun State, NIGERIA.

\begin{abstract}
In this study, we took advantage of the emergence of accurate biomechanical human hand models to develop a system in which the interaction between a human arm and a rehabilitation robot while performing a planar trajectory tracking task can be simulated. Seven biomechanical arm models were based on the 11-degree-of-freedom Dynamic Arm Simulation model and implemented in OpenSim. The model of the robot was developed in MatlabSimulink and interaction between the arm and robot models was achieved using the OpenSim API. The models were tested by simulating the performance of each model while moving the end effector of a simulated planar robot model through an elliptical trajectory with an eccentricity of 0.94. Without assistance from the robot, the average root-mean-square error (RMSE) for all subjects was $3.98 \mathrm{~mm}$. With the simulated robot providing assistive torque, the average RMSE error reduced to $2.88 \mathrm{~mm}$. The test was repeated after modifying the length of the robot links, and an average RMSE of $2.91 \mathrm{~mm}$ recorded. A single-factor ANOVA test revealed that there was no significant difference in the RMSE for the two different robot geometries ( $p$-value $=0.479$ ), revealing that the simulator was not sensitive to robot geometry.
\end{abstract}

Keywords: rehabilitation robotics, control, modelling, simulation, biomechanical models, OpenSim

\section{INTRODUCTION}

The increased interest in post-stroke rehabilitation robotics over the last two decades is largely due to its potential advantages over conventional rehabilitation therapy. Post-stroke functional outcomes are strongly mediated by exercise intensity, task specificity, active engagement and focusing on motor coordination [1] making rehabilitation very labour-intensive. These are all specifications for which properly-designed robots could reasonably be expected to be at least as effective as conventional therapy. In addition, shortage of therapists, difficulty in achieving cooperative and intensive efforts from therapists and patients over multiple sessions, and subjective evaluation methods are challenges with conventional therapy that would be minimized with robots. While

\footnotetext{
* Corresponding author (Tel: +234 (0)703 070 5929)

Email addresses: kayodele@oauife.edu.ng (K. P. Ayodele), akinniyioluwasegun@gmail.com (O. T. Akinniyi), oluwatopel@gmail.com (A. O. Oluwatope), jubrila@oauife.edu.ng (A. M. Jubril),

abiolaogundele22@gmail.com (A. O. Ogundele), adeyoyinmak2000@yahoo.com (M. A. Komolafe)
}

there is insufficient evidence to declare the outright superiority of robotic training over traditional approaches, there is at least evidence that it improves motor impairment and strength [2]. Numerous studies have also shown that combining the two approaches leads to greater reduction in motor impairments and improvements in functional abilities ([3] for example).

There exists a large variety of rehabilitation robot designs in the literature, but almost without exception, they conform to the general structure laid out in Fig. 1. A training strategy links neuroscience and physiotherapy on one hand and robotics on the other. Variants of the task-specific training (TST) strategy are now preferred, but there are viable alternative training strategies [4]. The training strategy informs the control strategy, and ultimately the physical design of the robot. That physical design is usually dominated by a number of mechanical links which can utilize either an exoskeleton or end-effector form $[5,6]$. In both forms, their primary function is to provide either augmentative or resistive force or torque to the human user natural limb movements. This is 


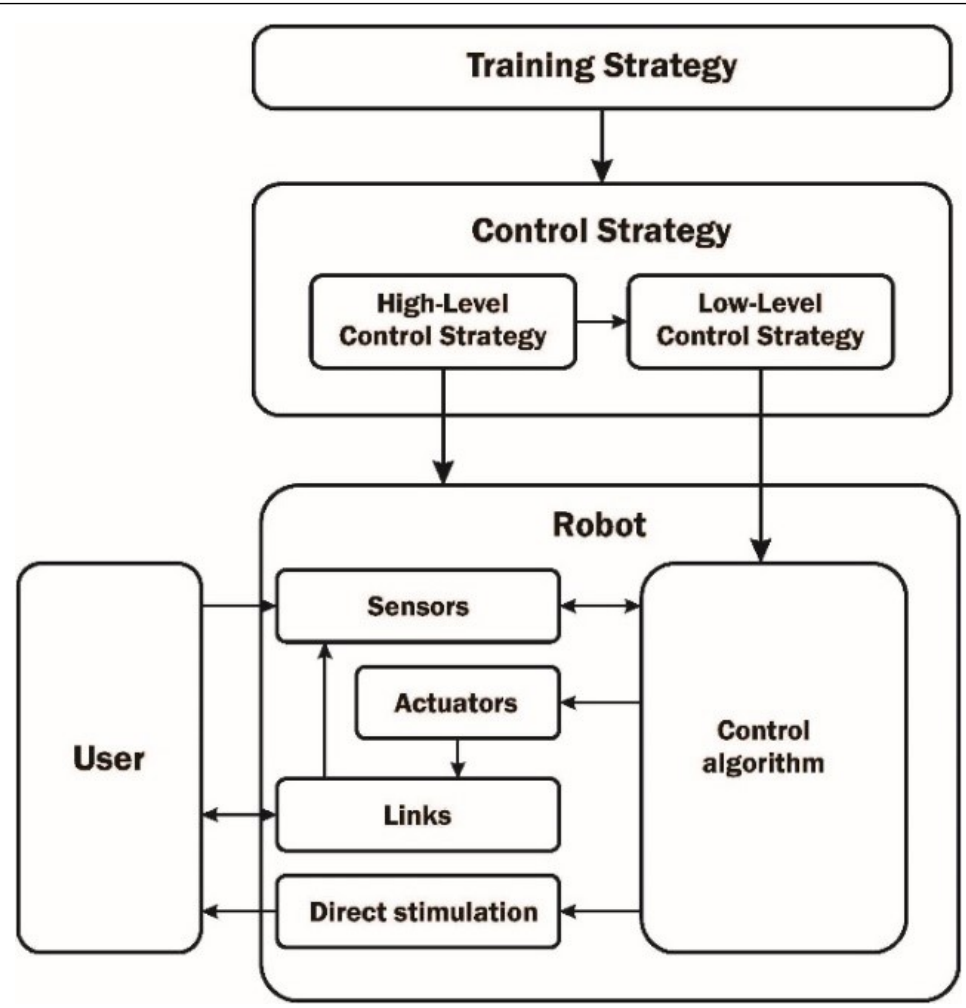

Figure 1: Generalized robotic rehabilitation elements.

most often achieved by means of electrical actuators, rather than pneumatic or hydraulic actuators. The convenience of easily integrating electrical actuators into systems that already have other electrical components usually outweigh the higher torque-to-weight ratio advantage of pneumatic actuators [5, 7]. Additional assistive torque may be generated by optional direct stimulation of the musculoskeletal or nervous system. Sensors acquire displacement, velocity or acceleration data or myoelectric or neurophysiological correlates from the user, while force or torque sensors quantify their effort.

Coordination of all rehabilitation robot system component is effected through a control algorithm dictated by the control strategy and implemented using an electronic controller. There are numerous control strategy taxonomies. Following the convention of [7], we adopt a classification into high and low level control strategies in this study. High level strategies include assistive control, challenge-based control, haptic control, and couching control strategies. The most commonlyused high level control strategy is currently the assistive one, in which patients are assisted to move their affected limbs in a stipulated manner while carrying out activities like grasping, reaching, or walking [8]. The high level control strategy determines the low level control strategy which could be position control, adaptive position control, impedance control, admittance control, assistance-as-needed control or tunnel control, the last four of which are also collectively termed patient-cooperative control strategies [9].

The choice of control algorithm entirely de- termines the function of the robot vis-a-vis the adopted training strategy. A wrong control algorithm would reduce the effectiveness of the robot, regardless of the sophistication or ingenuity of the hardware elements. A case can therefore be made that the control algorithm is the most important aspect of the rehabilitation robot, and the ability to evaluate different alternative control laws would be desirable. Unfortunately, current development approaches usually make it difficult to evaluate multiple control algorithms for the same robotic hardware. Historically, there have been very few accurate human controller models. Consequently, control law development for robotic hardware usually requires involving a real human operator in a real control loop, preceded by an inconvenient and often time-consuming system identification process to tune system parameters. This limits the flexibility in terms of testing multiple control algorithms.

In this study, we took advantage of the emergence of accurate human neuromusculoskeletal (biomechanical) models to develop a system for simulating upper extremity rehabilitation with a robot, therefore providing a means by which multiple control algorithms can be tested while simulating the interactions with the human operator in the loop. The rest of this paper is organized as follows: Section 2 provides a rationale and methodology for developing normal and impaired biomechanical models, and then discusses a general modelling framework for simulating robothuman dynamics. In Section 3, we present a detailed implementation example of the use of the simulator to test a five-link planar arm rehabilita- 
tion robot operating in assistive mode to help the subject trace a trajectory. Results are presented in Section 4, with concluding remarks coming in Section 5.

\section{MATERIALS AND METHODS}

The existence of accurate dynamic models of a plant makes it easier to design controllers to meet desired performance objectives. For the inverse dynamics control approach in particular, knowing the dynamics of the plant to be controlled allows it to be inverted, rapidly leading to a control law which cancels out the nonlinear aspects of the dynamics, decouples interactions between regulated variables and allows easy specification of the time characteristics of the delay of task errors [10]. Even for other controller design approaches, knowledge of at least the general form of the plant is useful. It is therefore very convenient that rehabilitation robots are usually structurally simple, with easily derivable kinematic and dynamic models which can be completely defined with direct or indirect dynamic parameter identification.

Consequently, the development of a rehabilitation robot control system usually involves the formulation of the robot dynamic model along with some sort of dynamic parameter identification process. In order to effectively optimize the controller, it is often necessary to adopt a model reflecting the dynamical properties of the human operator. Most rehabilitation robots employ assistive control strategies and for them, the expected movements of the human operator and the robot are defined beforehand, minimizing the role of human cognition and volition in the interaction with the robot, and simplifying the dynamics of the human operator within the control system.

In order to develop a simulator for testing rehabilitation robot control algorithms, it is necessary to first develop models of the human operator, and introduce elements capable of sufficiently capturing the dynamics of human-robot interaction. Hence, this section first discusses the development of upper limb extremity dynamic models for both normal and impaired human operators, and subsequently discusses how those models can be integrated with models of planar robotics arms in simulations that expose the salient points of their interactions. A detailed implementation example is thereafter given to illustrated how the system can be used to simulate a particular planar robot and evaluate how different control algorithms would work on it.

\subsection{Normal and Impaired Arm Biomechani- cal Models}

There are a number of software tools for human neuromusculoskeletal modelling and simulation. They include commercial packages such as AnyBody [11], MSMS [12], SIMM [13] and free, open source packages like OpenSim [14]. OpenSim was adopted in this study because apart from being free, its open, extensible architecture allows seamless integration with computational packages such as Matlab-Simulink. OpenSim models can be defined or edited graphically within the programme GUI, programmatically using any of a number of programming language options, or by directly generating a model text file. This flexibility allows easy re-use of OpenSim models. All the models used in this study were derived from the Dynamic Arm Simulation (DAS) biomechanical model [15]. The DAS model is a complex 3D model of the upper limb that is fast enough to run in real time on a personal computer. A base model was derived from the DAS model, and four variants of the base were generated as described below.

The base model was generated using anthropomorphic measures obtained from the cadaver of an adult male, and included the muscles and bones of the thorax and right hand. There are a total of 138 musculotendon actuators in 29 muscle groups, and 13 joints. The model has 11 degrees of freedom (DOF): 3 each at the glenohumeral, sternoclavicular and acromioclavicular joints, one for elbow flexion/extension and the last for forearm pronation/supination.

Human arm motion is the result of contractions in antagonistic muscles pairs under the influence of neural excitations. Choice of muscle model is fundamental to an accurate biomechanical model. We adopted a Hill-type [16] muscle model for the generation of muscle force from activation signals. In this model, the active force generation for a muscle with length $L_{M}$ is achieved through a contractile element (CE) of length $L_{C E}$. Tendons and other series stiffnesses are represented by a series elastic element (SE), while a parallel elastic element (PE) captures the muscle belly passive stiffness. The pennation angle $\alpha$ is the angle between the longitudinal axis of the muscle mass and its constituent fibres. Increased tension in the fibres results in an increase in the pennation angle and a reduction in the force transmitted to the tendon.

We modelled PE and SE as nonlinear springs in which element length, $L$, and generated force, $F_{K E}$ are related as follows:

$$
F_{K E}= \begin{cases}k_{1}\left(L-L_{s}\right) & \mid L \leq L_{s} \\ k_{1}\left(L-L_{s}\right)+k_{2}\left(L-L_{s}\right)^{2} & \mid L>L_{s}\end{cases}
$$

where $L_{s}$ and $k_{2}$ are constants whose values depends on the element type, and $k_{1}$ is a positivevalued constant. The positive value of $k_{1}(10 \mathrm{~N} / \mathrm{m}$ in this study) prevents a singularity from being formed in the Jacobian of the system dynamics at zero activation. The constant $k_{2}$ for the SE was set at a value such that the SE force at $4 \%$ elongation equalled the maximum isometric force. For the $\mathrm{PE}$, the value of $k_{2}$ was that which allows the $\mathrm{PE}$ force to equal the maximum isometric force when the $\mathrm{CE}$ was stretched to its maximum length for active force production. The relationship between muscle active state, $a$, and the neural excitation $\mathrm{u}$, was modelled as $[15,17]$ : 


$$
\dot{a}(t)=\left(\frac{u(t)}{T_{a c t}}+\frac{1-u(t)}{T_{\text {deact }}}\right)\{u(t)-a(t)\}
$$

where $T_{a c t}$ and $T_{\text {deact }}$ are the activation and deactivation time constants respectively. In accordance with [18], the values of $T_{\text {act }}$ and $T_{\text {deact }}$ were estimated from the proportions of fast and slow twitch fibres in the muscle. Muscle active state, $a$ and the force $F_{C E}$ generated by the CE are related as follows:

$$
F_{C E}=a F_{\max } f_{F L} f_{F V}
$$

where $F_{\max }$ is the maximum isometric force the muscle is capable of generating, $f_{F L}$ is the isometric force-length relationship of the $\mathrm{CE}$, and $f_{F V}$ is the force-velocity relationship of the $\mathrm{CE}$. In this study, the following approximations were adopted for $f_{F L}$ and $f_{F V}[15]$ :

$$
\begin{gathered}
f_{F L}=\exp ^{-\left(\frac{L_{C E}-L_{C E O}}{W \cdot L_{C E O}}\right)^{2}} \\
f_{F V}= \begin{cases}\frac{V_{\max }+V_{C E}}{V_{\max }-\frac{V_{C E}}{A}} & V_{C E} \leq 0 \\
\frac{g_{\max } V_{C E}+c_{3}}{V_{C E}+c_{3}} & V_{C E}>0\end{cases}
\end{gathered}
$$

where $L_{C E}$ is the contrative element length, $L_{C E O}$ is a muscle-dependent constant termed the optimal CE length, and $\mathrm{W}$ is the parameter of the force-length curve. We set the value of $\mathrm{W}$ as 0.56. $V_{C E}$ is the velocity of the CE element, $V_{\max }$ is the maximum shortening velocity at full activation and $A$ is a constant. $g_{\max }$ is the maximal normalized eccentric muscle force, and $c_{3}$ is a muscle-dependent constant. We used values of $0.25,10 L_{C E 0}$, and 1.5 for $A, V_{\max }$ and $g_{\max }$ respectively, and set $c_{3}$ to a value that generates a continuous first derivative when $V_{C E}=0$.

The base model has 22 states representing 11 angular displacements and 11 angular velocities. We adopted the following equation of motion:

$$
M(q) \ddot{q}+B(q, \dot{q})+C(q) \cdot \Psi=0
$$

in which $q, \dot{q}, \ddot{q} \in \mathbb{R}^{11}$ are the vectors of joint displacement, velocity and aceleration respectively, $M, B, \in \mathbb{R}^{11 \times 11}$ are inertial and coriolis matrices which account for muscular inetia and Coriolis forces along with ligament and contact forces respectively. $\Psi$ is the vector of joint and muscle moments, and $C \in \mathbb{R}^{11}$ is the coefficient for accounting for the effect of muscle moments.

To adequately simulate the interactions between the rehabilitation robot and stroke survivors with different movement abnormalities, it is necessary to introduce abnormal dynamics into the arm model. The cover-all term for the positive and negative motor symptoms that occur after stroke is "hemiparesis". A number of studies have attempted to identify distinct categories of hemiparetic deficit. One of the most notable studies [19] identified the following categories of deficits: muscle weakness, spasticity, loss of muscle synergies, and a deficit in feed-forward compensation for interaction torques, exhibited as a loss of joint coordination resulting from an inability to anticipate the effect of shoulder acceleration on acceleration at the elbow.

Muscle weakness is a common stroke symptom which manifests as a reduction in the contraction strength of affected muscles, and consequently, impairment in completion of the activities mediated by the muscles, such as gripping. We modelled muscle weakness as a reduction in the maximum isometric forces of muscles [20]. For weak muscles, Eq. (1) was modified by the introduction of a factor, $\mu$ so that:

$$
F_{C E}=a \mu F_{\text {max }} f_{F L} f_{F V}
$$

We defined three levels of weakness, namely mild, moderate, and severe with $\mu$ values of 0.250 , 0.125 , and 0.0625 respectively [21]. The forcelength and force-velocity curves of the Hill-type muscle model were scaled accordingly for each level of weakness. The weakness correction was uniformly applied to every muscle in a muscle group.

Spasticity has been defined as a velocitydependent increase in resistance against externally imposed joint movements [22] that is characterized by exaggerated stretch reflex. However, biomechanical models of spasticity based on velocity alone fail to capture certain salient features of spastic response, such as the oscillations in measured EMG activity or sustained muscle activity following a stretch [23]. We adopted a stretch reflex activation model based on stretch velocity and muscle length, which also factors in the relative proportions of slow and fast twitch fibres [24]. In this model, the activation generated by a muscle experiencing stretching is given by

$$
a(t)=\gamma\left\{\frac{w_{S}}{\lambda_{L} l_{0}^{m}}\left(L_{F}-\lambda_{L} l_{0}^{m}\right)+\frac{w_{F}}{\lambda_{V} V_{\max }}\left(\dot{L}_{F}-\lambda_{V} V_{\max }\right)\right\}
$$

where $\gamma$ is an adjustable gain parameter, $w_{S}$ and $w_{F}$ are weighting factors of slow and fast twitch fibres respectively such that $w_{S}+w_{F}=1 . L_{0}^{m}$ is the optimal muscle length, $\lambda_{L}$ and $\lambda_{V}$ are muscledependent parameters, and $V_{\text {max }}$ is the maximum muscle stretch velocity. Force generation in the spastic muscle is then computed using Eq. (1) and (3). In the upper extremities, spasticity is usually experienced in the flexor muscles, although exceptions are known [25].

Abnormal muscle synergy is a well-known sequela of stroke. Muscle synergies are vectors specifying patterns of relative muscle activation levels. They are believed to be adaptations that allow task-level commands to be translated into execution-level activation patterns, mirroring the hierarchal structure of multisensory integration systems [26]. In normal vertebrate physiology, the nervous system adaptively activates sequences of muscle co-activations with simple neural commands. This increases the informational efficiency of the nervous system. When 
the controlling neuronal populations are affected by a lesion however, the normal synergies may be disrupted, leading to stereotyped abnormal coactivation of multiple muscle groups that reflect a loss of independent joint control, which limits the patient's ability to coordinate their joints in creating arbitrary movement patterns [27].

We modelled two well-known abnormal poststroke forearm synergies, namely flexor synergy (in which elbow flexion is accompanied by shoulder abduction and forearm supination) and extension synergy (involving simultaneous elbow extension, shoulder adduction, and forearm pronation) [28]. The muscle activation for muscles recruited into abnormal sympathetic movement during flexion or extension is:

$a_{s y n}^{*}= \begin{cases}a_{s y n}+\zeta a_{m}\left(\frac{\phi-\phi_{\min }}{\phi_{\max }-\phi_{\min }}\right) & \text { if } \phi_{\min } \leq \phi<\frac{\phi_{\max }-\phi_{\min }}{2} \\ a_{s y n}+\zeta a_{m}\left(\frac{\phi_{\max }-\phi}{\phi_{\max }-\phi_{\min }}\right) & \text { if } \frac{\phi_{\max }-\phi_{\min }}{2} \leq \phi<\phi_{\max }\end{cases}$

where $a_{s y n}$ is the original activation of the sympathetic muscle, $a_{s y n}^{*}$ is the adjusted activation, $a_{m}$ is the activation of the biceps for flexor synergy, or the triceps for extensor synergy. $\zeta$ is a gain factor for which we used the value 0.1 , while $\phi_{\min }$ and $\phi_{\max }$ are threshold elbow flexion angles between which abnormal synegy would kick in. Muscle groups sympathetically activated during flexor synergy include supinator, deltoid and supraspinatus. For extensor synergy, pronator teres, pronator quadratus, pectoralis major, latissimus dorsi, teres major, and coracobrachialis were activated in sympathy with the activation on the triceps.

\subsection{Simulation Framework}

In a normal arm rehabilitation session using an end effector robot and a tracking task, the robot exerts a vector of torques and forces on the patient's arm (Fig. 2). This vector combines with actuation from muscles to move the patient's arm in an attempt to follow the prescribed path. The human brain controls the sensory and executive functions required to generate the proper activation for muscles. Although the complexities or, and variation in human anatomy and physiology ordinarily precludes accurate models of the human operator, a trajectory tracking task minimizes the role of human volition and simplifies the modelling requirements. Once the current location of the end effector is known, the desired next location can be predicted with very high accuracy, which means that the actions of the real normal human operator can be predicted with comparable accuracy, subject to minor random errors.

Let us assume a specified trajectory $\chi=$ $\chi_{i}(\mathbf{T}, \mathbf{F}, \boldsymbol{\theta}, i=1,2 \ldots, N$, where the iterator $i$ indicates the current position in the sequence of $N$ states, and the trajectory has been parameterized in terms of vectors of torques (T), forces $(\mathbf{F})$ and angular displacements $(\boldsymbol{\theta})$. The movement of a point of interest on the human arm can similarly be parameterized $\mathbf{M}=M_{i}(\mathbf{T}, \mathbf{F}, \boldsymbol{\theta}, i=1,2 \ldots, N$, . The goal of a tracking task is to find the optimum dynamical sequence $M_{o p t}$ for the point of interest on the arm using some objective function, $J(\cdot)$ such that

$$
M_{o p t}=\arg _{\mathbf{M}}^{\min } \sum_{i=1}^{N} j\left(\chi_{i} M_{i}\right)
$$

where $\chi_{i}$ and $M_{i}$ are the previously defined desired and arm trajectories. To achieve this, a real human applies feedback and an integrative process to determine the proper amount of force or torque to apply in order to arrive at the desired next end effector location. Using sensory-motor cooperation learned over years, the nervous system automatically supplies the appropriate neural excitations and muscle activation to generate the required forces and torques. In fact, the motor deficits stroke sequelae arise from the failure of this process, due to impairments in either excitation mechanism within neuronal populations, or nervous system pathways. A biomechanical model within a simulated rehabilitation block must be able to generate the requisite forces and torques for a normal user, and also introduce realistic failure modes that correspond to real hemiplegic deficits. We did this, and employed a predictor-corrector scheme to integrate the dynamics of the robotic arm. First, a predictor step was used to generate the future state based on only the dynamics of the human model.

$$
M_{i+\frac{1}{2}}=F\left(M_{i}, \chi_{i}\right)
$$

Given the noise in both normal and abnormal human models, some error existed between this predicted next state and the next state in the trajectory. The robot controller therefore operated on this error in a corrector step:

$$
M_{i}=f\left(M_{i+\frac{1}{2}}, j\left(\chi_{i+1}, M_{i+\frac{1}{2}}\right)\right)
$$

where, as before, $j\left(\chi_{i+1}, M_{i+\frac{1}{2}}\right)$ is an objective function, and the nature of the function $f(\cdot)$ depends on the low-level control strategy. An example using impedance control is considered in Section 3.

Our simulation framework therefore carried out the following steps : first, the next desired location $\chi_{i}(\mathbf{T}, \mathbf{F}, \boldsymbol{\theta})$ in the trajectory was obtained, allowing the internal joint configuration in the arm model to be determined by inverse kinematics. Thereafter, the internal forces and torques about joints was computed using an inverse dynamic optimization. Using a static optimization procedure, these internal forces and torques were used to compute the required muscle activations, which were then modified to add noise whose nature depended on the absence or nature of hemiplegia being modelled. Thereupon, forward dynamics was executed to determine the predictor next position. This was then operated upon by the robot model in concert with the applicable control algorithm to obtain the corrected next position. 


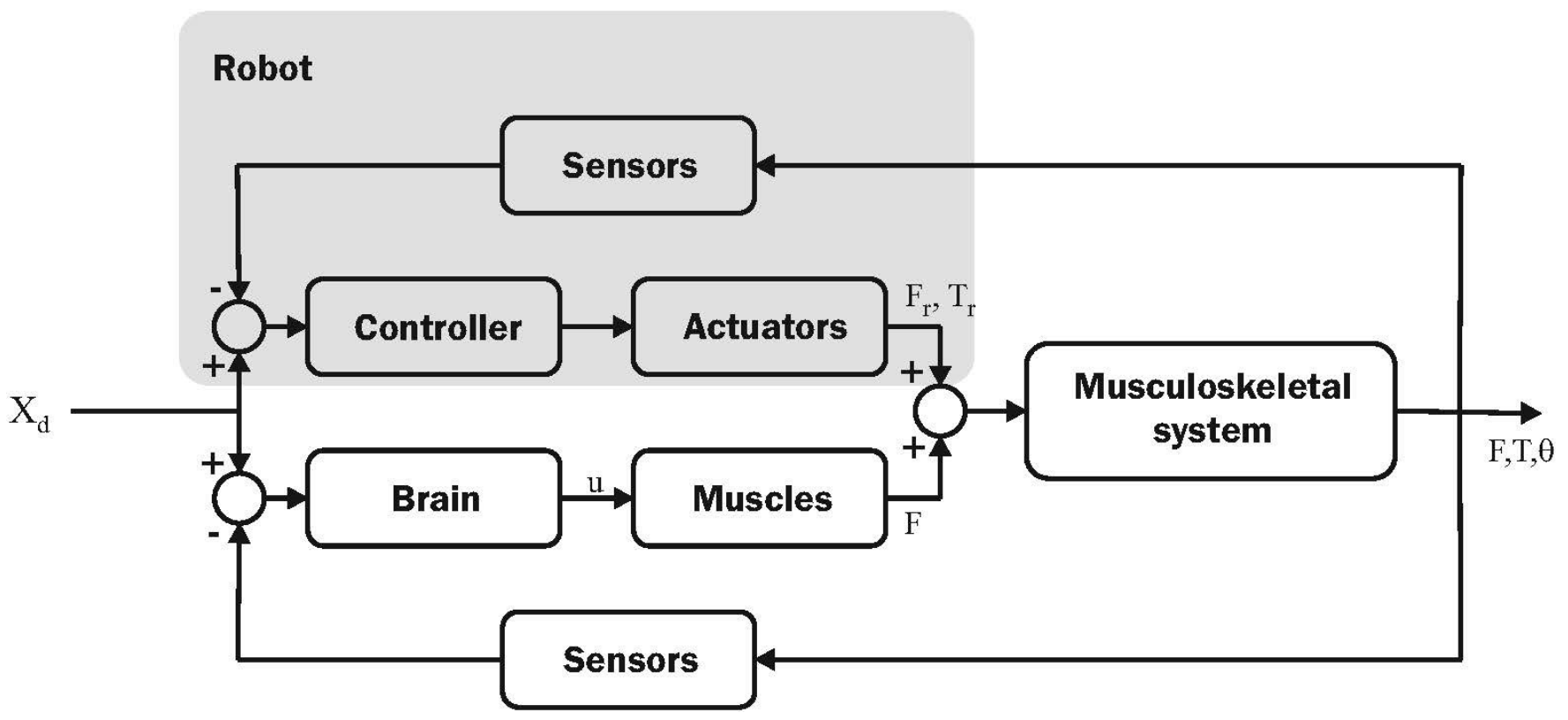

Figure 2: Block diagram showing human and robotic components during rehabilitation.

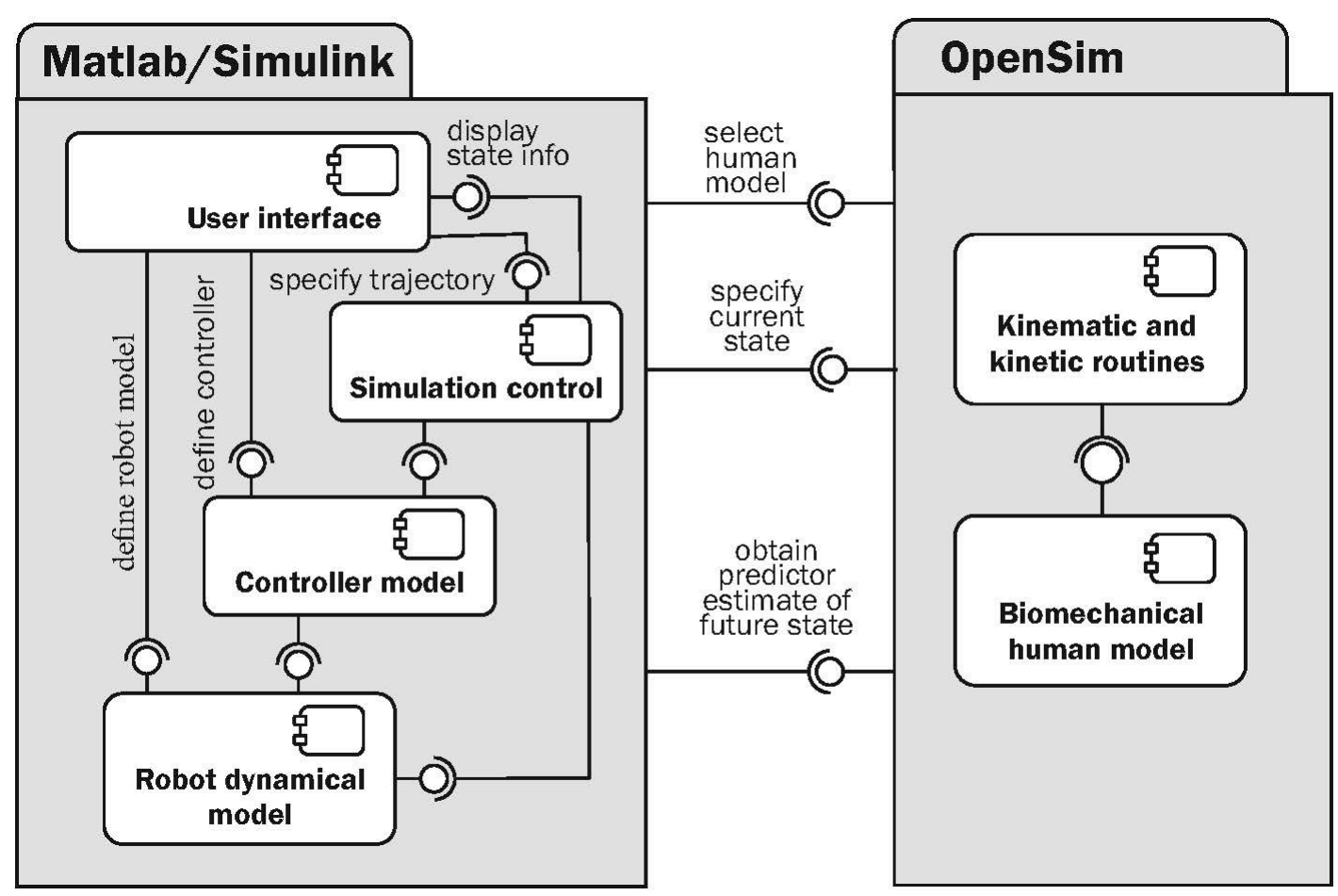

Figure 3: Component diagram of the simulation framework. 
The goal of the inverse kinematics step was to determine the set of joint angles that best matched the next specified location in the trajectory. This required the specification of the intended location as markers, and the solving of a weighted elast squares optimization problem while minimizing the error between required and computed marker positions. We associated each marker with a weight which indicated how strongly the marker's error term should be minimized in the least squares optimization problem. The operation of the inverse kinematic step is given by

$$
\min _{q}\left[\sum_{i \in \text { markers }} w_{i}\left\|x_{i}^{\exp }-x_{i}(q)\right\|^{2}\right]
$$

Inverse dynamics computation thereafter allows the determination of the net forces and torques at all the joints that would give rise to the movement obtained in the inverse kinematics step. We used Eq. (6) in which displacement was obtained from inverse kinematics and velocity determined with inverse kinematics and next trajectory state, the vector of all joint moments $\Psi$ was computed. in the static optimization step, the known motion of the model was then used to solve the equation of motion for unknown generalized forces subject to ideal force generators.

The simulation framework is presented in Fig. 3 in the form of a component diagram. Interaction between Matlab and OpenSim was through the OpenSim Matlab API. To simulate a rehabilitation session, a specific human model was seected. A trajectory was formulated and transferred to the shared coordinate system. The dynamic model of the robot was developed, after which the control algorithm to be tested was inserted. it should be noted that within this framework, the biomechanical human model played the role of real human; it can therefore be referred to as a virtual human. Depending on the control law being tested, the controller may integrate a simpler model of the human arm, and indeed system identification may be carried out, with the virtual human serving in lieu of a real one.

\section{IMPLEMENTATION}

\subsection{Description of Robot}

We used an implementation of the simulation framework for testing control algorithms for the PULSR parallelogram arm rehabilitation robot of Obafemi Awolowo University, Nigeria (Fig. 4). The robot has five links with four of them configured as a standard parallelogram arm while the fifth link serves as a mount for the patient forearm. Links are fabricated out of $5.08 \mathrm{~cm}$ wide extruded 6061 aluminium bars with rolling ball bearing joints. Two $60 \mathrm{~W} 24 \mathrm{~V}$ NEMA 23 brushless DC (BLDC) servomotors coaxially mounted at point A actuate link 1 and link 2. Each motor is capable of generating $12.5 \mathrm{Nm}$ continuous torque and $35 \mathrm{Nm}$ peak torque using a 100:1 planetary gearbox. Three two-phase 600 pulses/revolution incremental rotary encoders at points $\mathrm{A}$ and $\mathrm{B}$ allow the angular configuration of the robot to be determined. The forces generated by the user as they use the robot are determined by means of a 3 -axis force sensor installed at the base of the end effector at point B. A 4-channel functional electrical stimulation (FES) amplifier is used to generate pulse width modulated signals for application to the triceps of the subject. Graphite-and-steelball air bearing ensures minimal friction between the robot and workspace table.

During normal operation, a subject's forearm is strapped to link 5, with the subject grabbing the end effector located at $B$, and attempting to move it to trace a pre-specified elliptical trajectory at constant velocity. The predefined trajectories are projected from an overhead projection system, with multiple trajectories possible using workstation. For this study, an elliptical trajectory with eccentricity of 0.94 was used. The user is firmly strapped to the high backrest of their seat using padded straps in order to minimize movement of the acromioclavicular and sternoclavicular joints. FES signals may be applied to the arm undergoing rehabilitation. Muscle weaknesses and spasticity are two common stroke symptoms for which FES is commonly applied. Most stroke patients will eexperience problems with elbow and shoulder extension during reaching tasks at some point during recovery or rehabilitation. The application of pulse-width modulated FES signals to the triceps in particular can counteract this during rehabilitation. The FES input was not utilized in this study.

\subsection{Robot Dynamic Model and Control}

The geometry of the robot coupled with a human forearm is shown in Fig. 5 and the DenavitHartenberg parameters of the robot are presented in Table 1. The full length of Link 4 is $0.6 \mathrm{~m}$. The robot joint angle vector is $q_{r}=\left[\theta_{1} \theta_{2}\right]^{T}$ where $\theta_{1}$ and $\theta_{2}$ are the angles between the base frame and Link 1 and Link 2 respectively. The torque generated by the BLDC motors is represented by $\boldsymbol{\tau}_{r}=\left[\tau_{1} \tau_{2}\right]^{T}$. The configuration of the human arm is defined by $\mathbf{q}_{a}=\left[\theta_{f} \theta_{u}\right]^{T}$ where $\theta_{u}$ is the angle between the upper arm and a coronal plane passing through the glenohumeral joint, and $\theta_{f}$ is the angle between the projections of the forearm and the upper arm onto a transverse plane through the human body.

Table 1: Denavit-Hertenberg parameters for the parellogram arm robot.

\begin{tabular}{lllll}
\hline Link & $\mathbf{a}(\mathbf{m})$ & $\boldsymbol{\alpha}$ & $\mathbf{d}$ & $\boldsymbol{\theta}$ \\
\hline Link 1 & 0.3 & 0 & 0 & $\theta_{1}$ \\
Link 4 & 0.3 & 0 & 0 & $\theta_{4}=\theta_{2}-\theta_{1}$ \\
Link 3 & 0.3 & 0 & 0 & $\theta_{3}=\pi+\theta_{1}-\theta_{2}$ \\
Link 2 & 0.3 & 0 & 0 & $\theta_{2}$ \\
\hline
\end{tabular}

A form of impedance control was adopted for the interaction of the robot and the human operator. The primary advantage of impedance control 


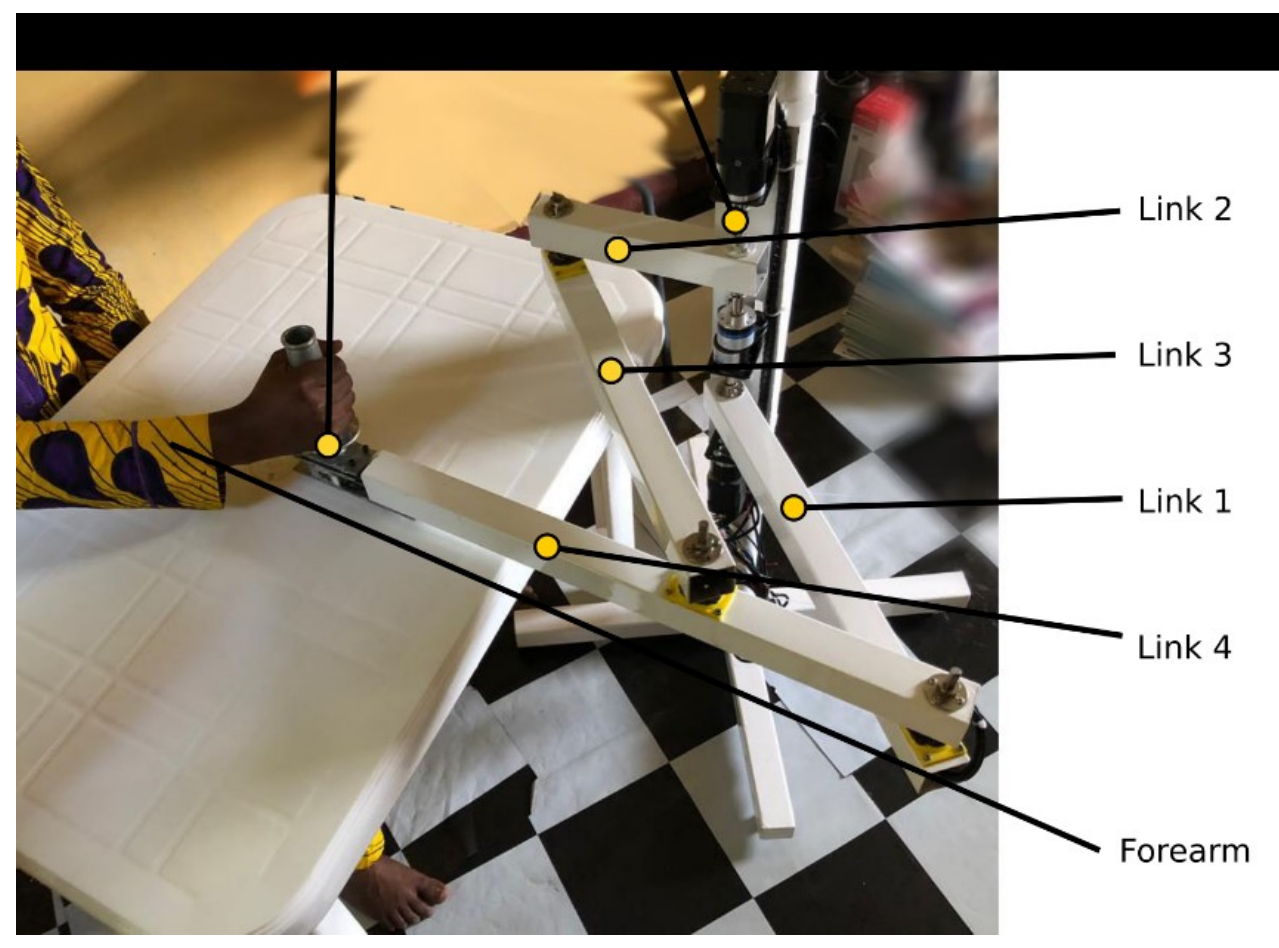

Figure 4: The real PULSR rehabilitation robot showing the 4 links.

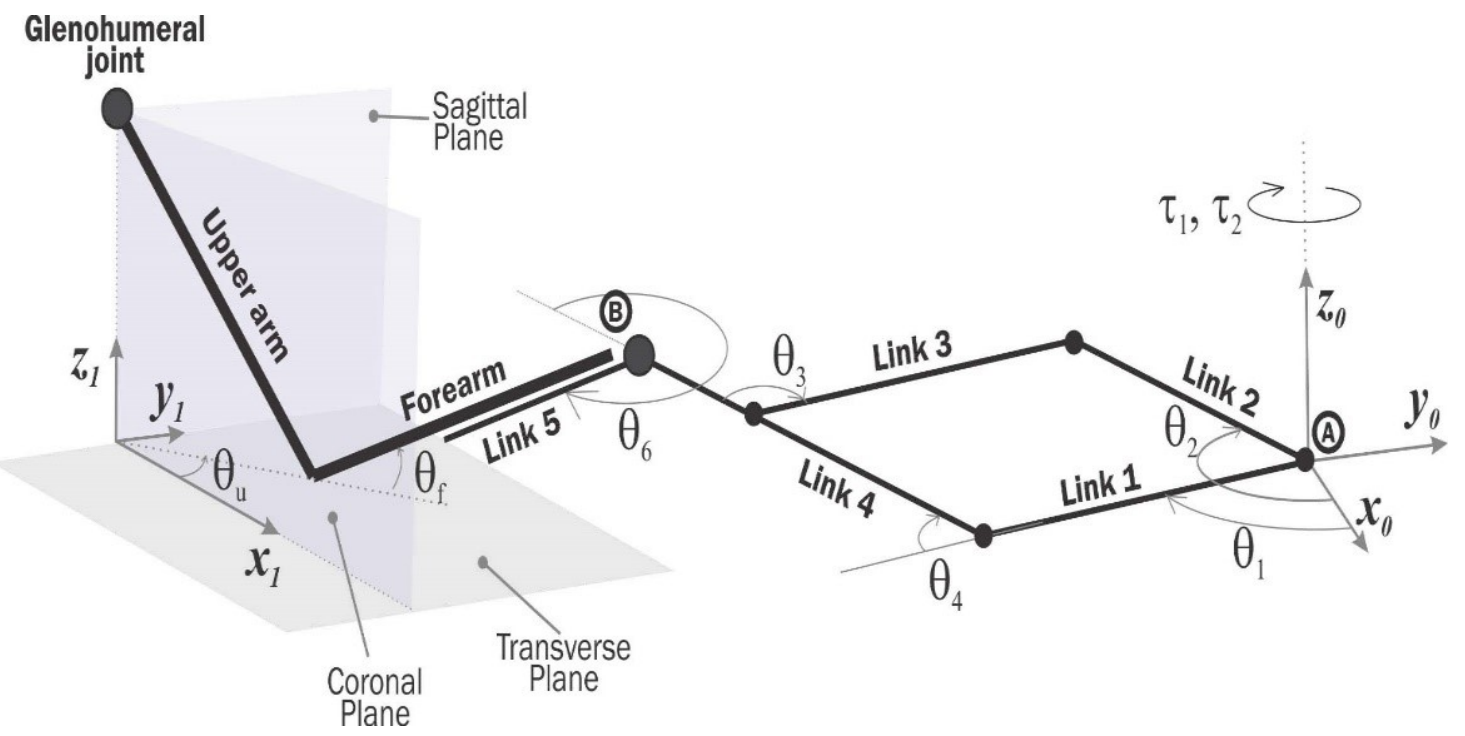

Figure 5: Geometry of the parallelogram arm robot showing a strapped human arm. 
is that it allows easy simultaneous control of motion and contact force. In so doing, it ensures the safety of the human operator from injuries caused by sudden large forces. The following relationship was assumed between robot displacement $\mathbf{q}_{r}$ and force $\mathbf{f}_{r}$ applied to the end effector at Point B [29]:

$$
\mathbf{f}_{r}=\mathbf{K}_{M} \ddot{\tilde{\mathbf{X}}}_{r}+\mathbf{K}_{B} \dot{\tilde{\mathbf{X}}}_{r}+\mathbf{K}_{K} \tilde{\mathbf{X}}_{r}
$$

where $\tilde{\mathbf{X}}_{r}=\mathbf{X}_{r e f}-\mathbf{X}_{r}$, in which $\mathbf{X}_{r e f}$ is the reference position and $\mathbf{X}_{r e f}=\mathbf{k}_{r}\left(\mathbf{q}_{r}\right)$. Also $\dot{\mathbf{X}}_{r}=\mathbf{J}_{r}\left(\mathbf{q}_{r}\right) \dot{\mathbf{q}}_{r}$ and $\mathbf{X}_{r}=\mathbf{J}_{r}\left(\mathbf{q}_{r}\right) \ddot{\mathbf{q}}_{r}+\dot{\mathbf{J}}_{r}\left(\mathbf{q}_{r}, \dot{\mathbf{q}}_{r}\right) \dot{\mathbf{q}}_{r} \cdot \dot{\mathbf{J}}_{r}^{T}\left(\mathbf{q}_{r}\right)$ is the Jacobian of the system and $\mathbf{K}_{M}, \mathbf{K}_{B}, \mathbf{K}_{K}$ are gain matrices whose value depends on the mode in which the robot is being operated of which there are three: "free-moving load" (FML), "tracking control" (TC), and "assistive torque" (AT). In the FML mode, the robot presents itself as a load that can be moved with ease by the user. Most interactions of human arms with their environment during normal day-to-day activities can be modelled as moving a point mass through a viscuous medium [18]. Therefore, in the FML mode, $\mathbf{K}_{K}=0$. Also, $\mathbf{K}_{M} K_{M} \mathbf{I}$ and $\mathbf{K}_{B}=K_{B} \mathbf{I}$, where $K_{M}$ and $K_{B}$ are real values that make the load feel "natural" to each user. In TC mode, the robot initializes the subject's arm at the beginning of each iteration of the task. The gain matrices are set as $\mathbf{K}_{M}=K_{M} \mathbf{I}$, $\mathbf{K}_{B}=K_{B} \mathbf{I}, \mathbf{K}_{K}=K_{K} \mathbf{I}$ with a further constraint that $K_{K}>0 . K_{M}$ and $K_{B}$ are tuned to achieve the required tracking performance. In the AT mode, the application of FES signals at the triceps generates assistive torque at the elbows. Since FES use was not considered in this study, the AT mode was not used.

\subsection{Trajectories and Testing}

In real life, gain matrices $\mathbf{K}_{M}, \mathbf{K}_{B}, \mathbf{K}_{K}$ are set during a preliminary system identification session in which they are tuned until operation of the robot feels "natural" to a user. Since the "user" in this study was a virtual user (the biomechanical model), we introduced a measure to replace what a normal user would have deemed "natural". According to [18], typical loads interacted with daily are most accurately modeled as a moving point mass through a viscuous medium. Motivated by typical values used in literature for real users, we selected values corresponsing to a load of $1.5 \mathrm{~kg}$ and viscuous friction of $30 \mathrm{~N} / \mathrm{ms}$. We thereafter generated ten trials each for an unimpaired virtual subject and five different impaired subjects using the simulink robot model in the FML mode. The root mean square error (RMSE) - calculated from the absolute value of the deviation between planned trajectory and the actual trajectory traced by the biomechanical model for each trial- was recorded. Subsequently, the same subjects were also simulated using the robot TC mode, and RMSE errors similarly recorded.

\section{RESULTS AND DISCUSSION}

The trajectories traced by the 7 subjects over a total of 60 trials are presented in Fig. 6 and 7. An

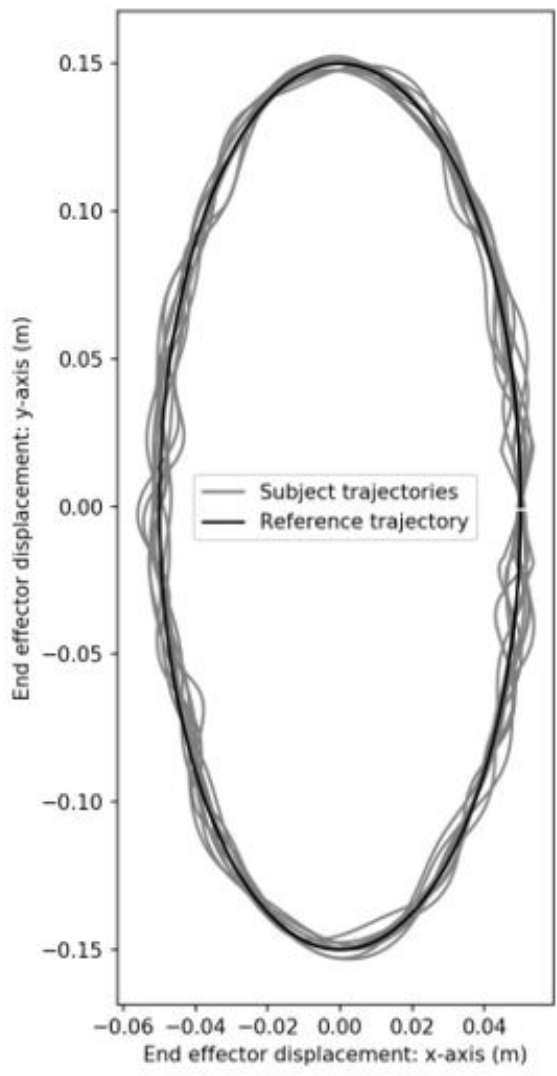

(a) simulated subject

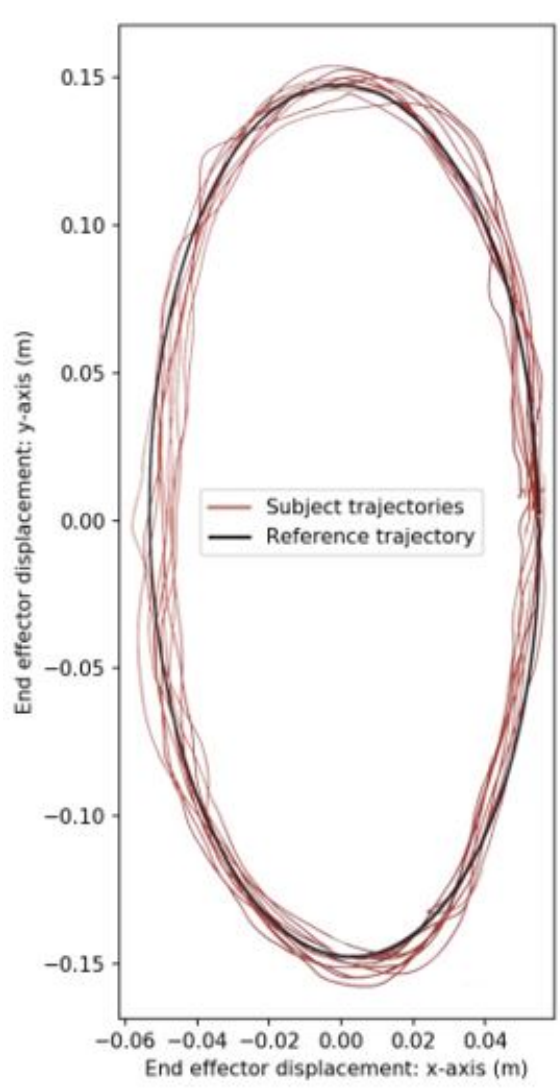

(b) real human subject

Figure 6: Trajectories resulting from 10 trial movements for unimpaired (a) simulated subject (b) real human subject. 


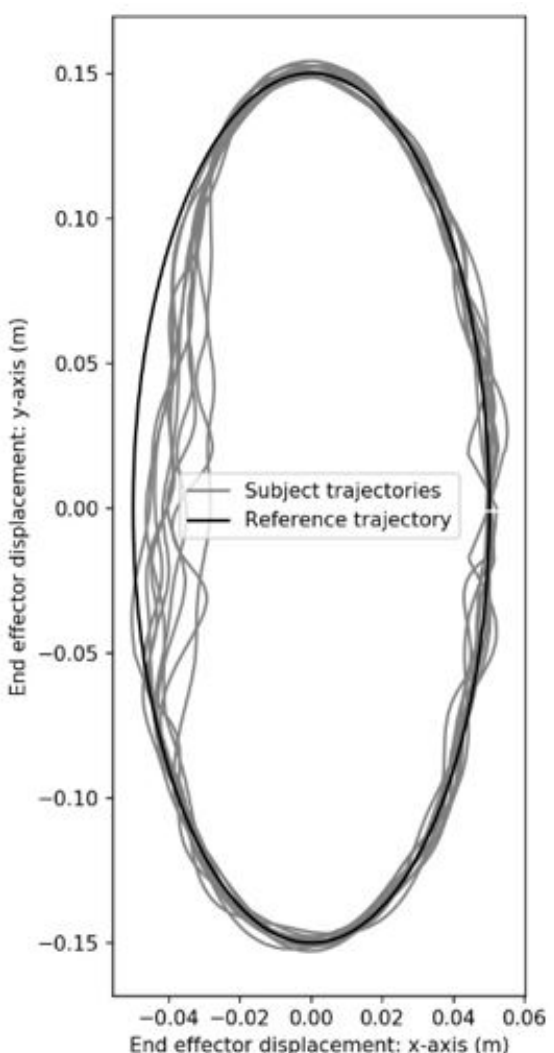

(a) flexion synergy

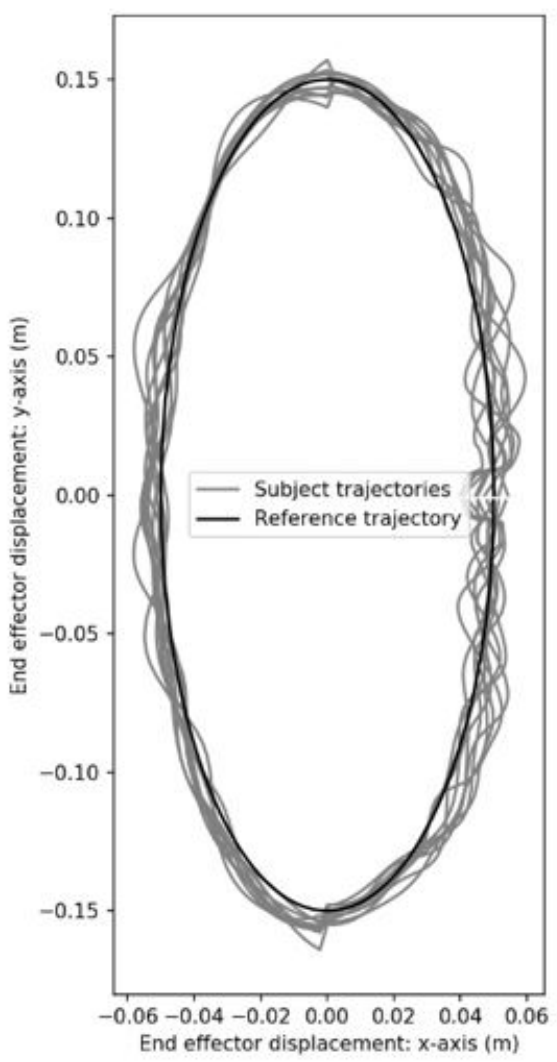

(c) spasticity

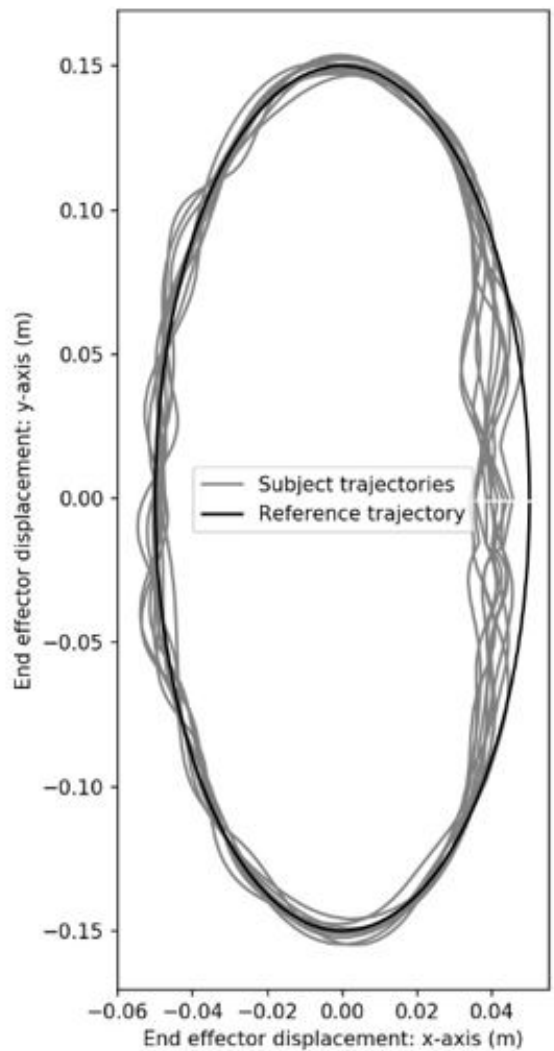

(b) extension synergy

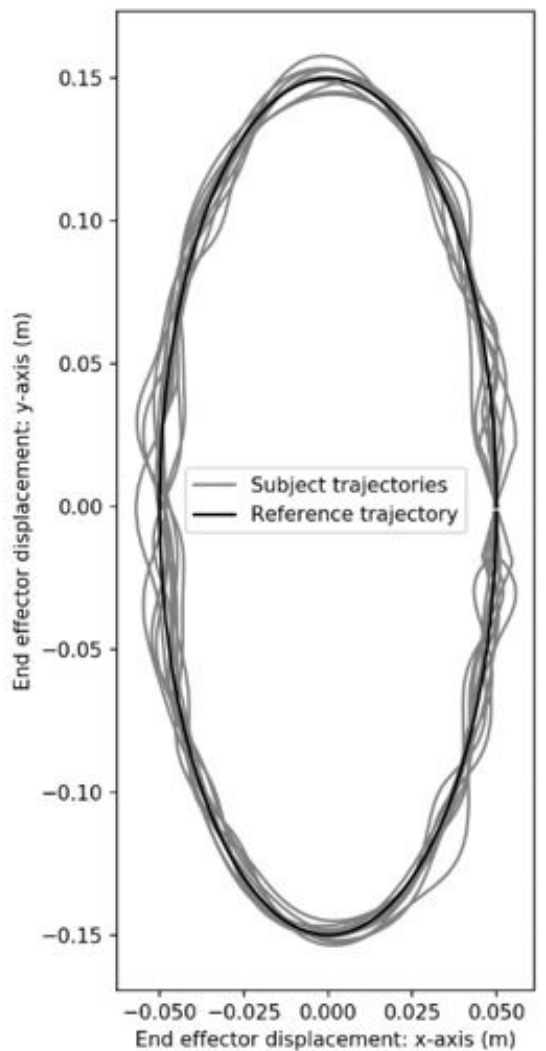

(d) muscle weakness

Figure 7: Trajectories resulting from 10 trial movements each for simulated subjects with the following impairments (a) flexion synergy (b) extension synergy (c) spasticity (d) muscle weakness. 


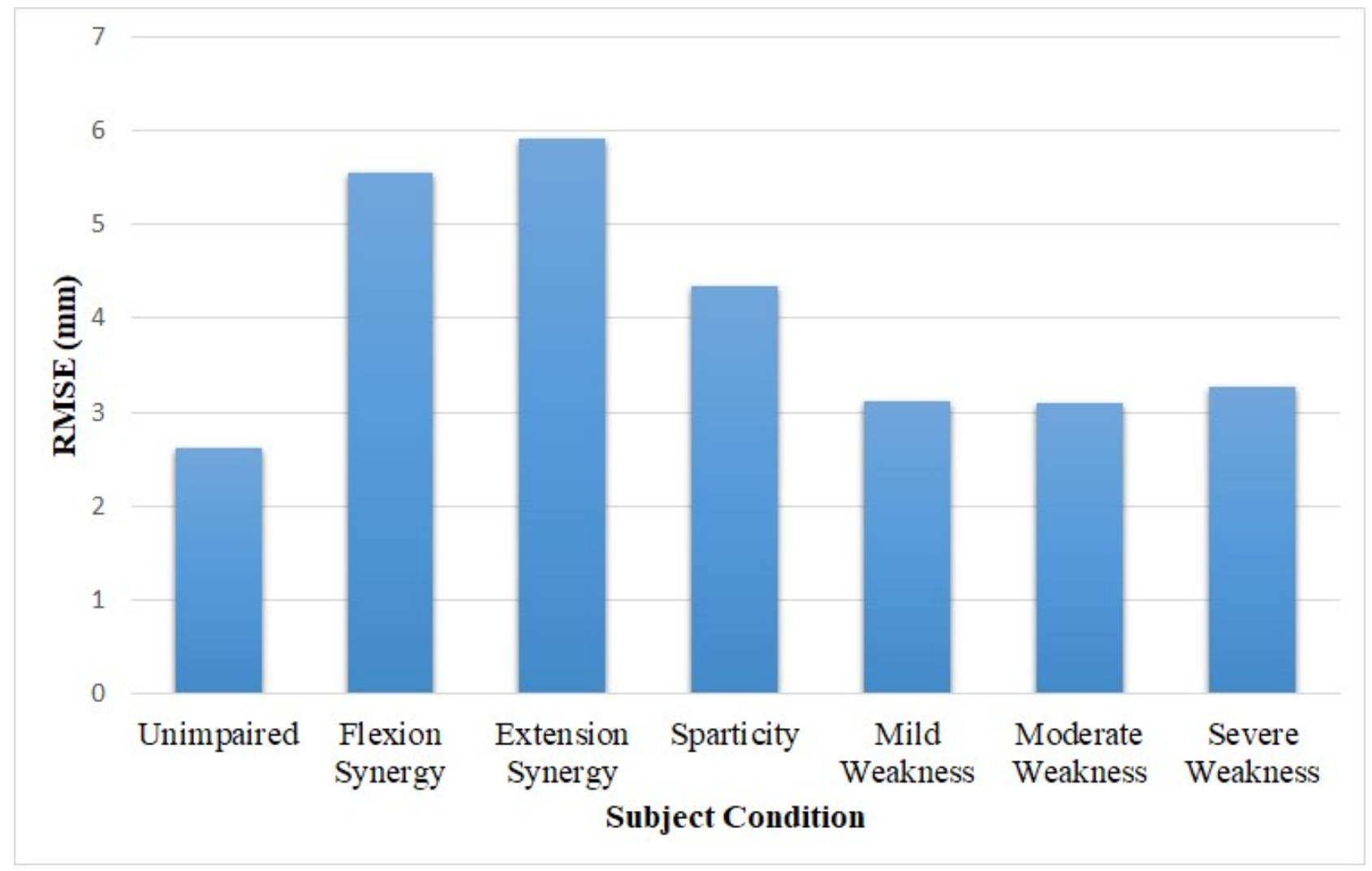

Figure 8: RMSE for the trajectories of 6 different simulated users in FML mode.

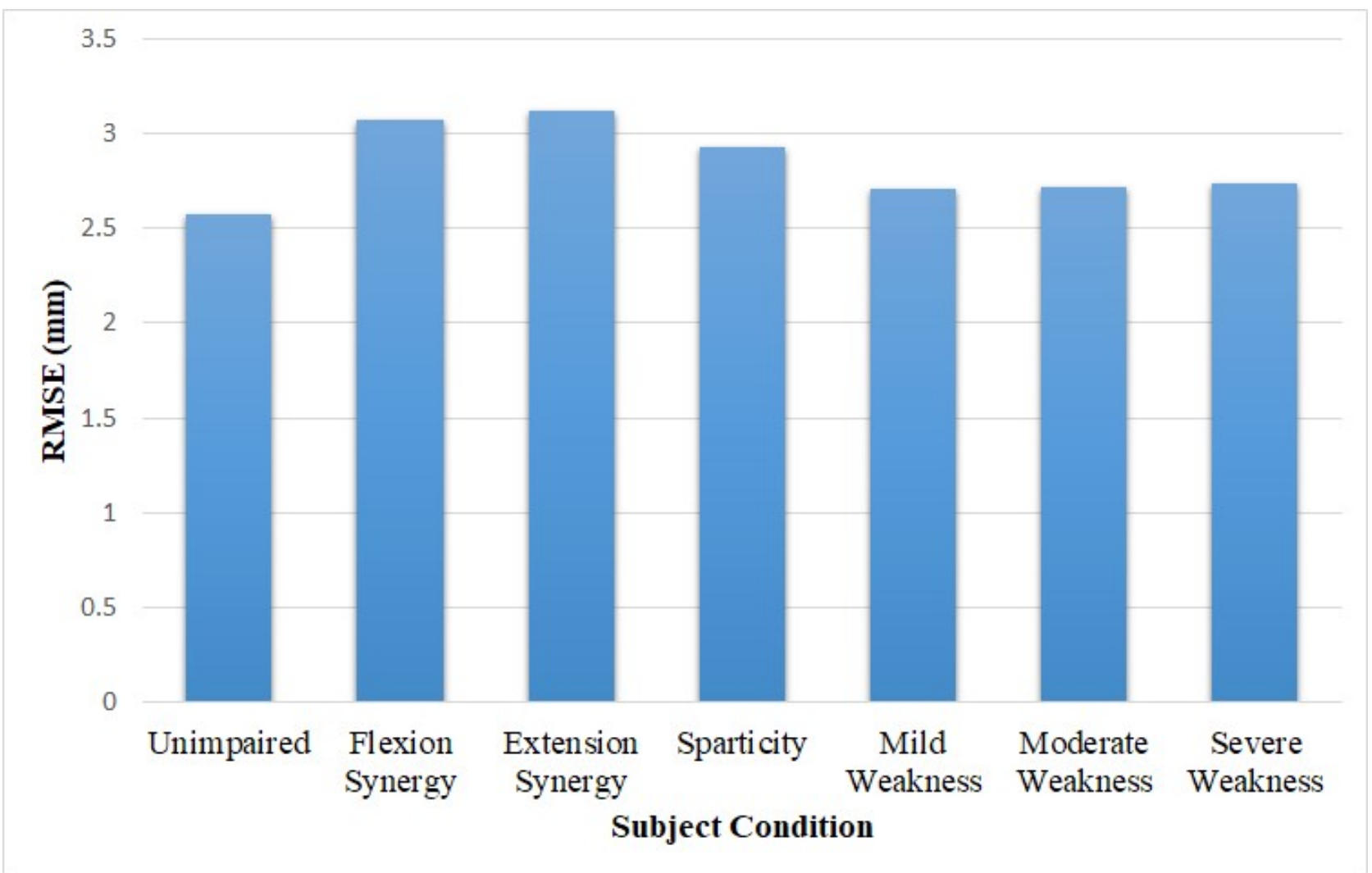

Figure 9: RMSE for the trajectories of 7 different simulated users in TC mode. 


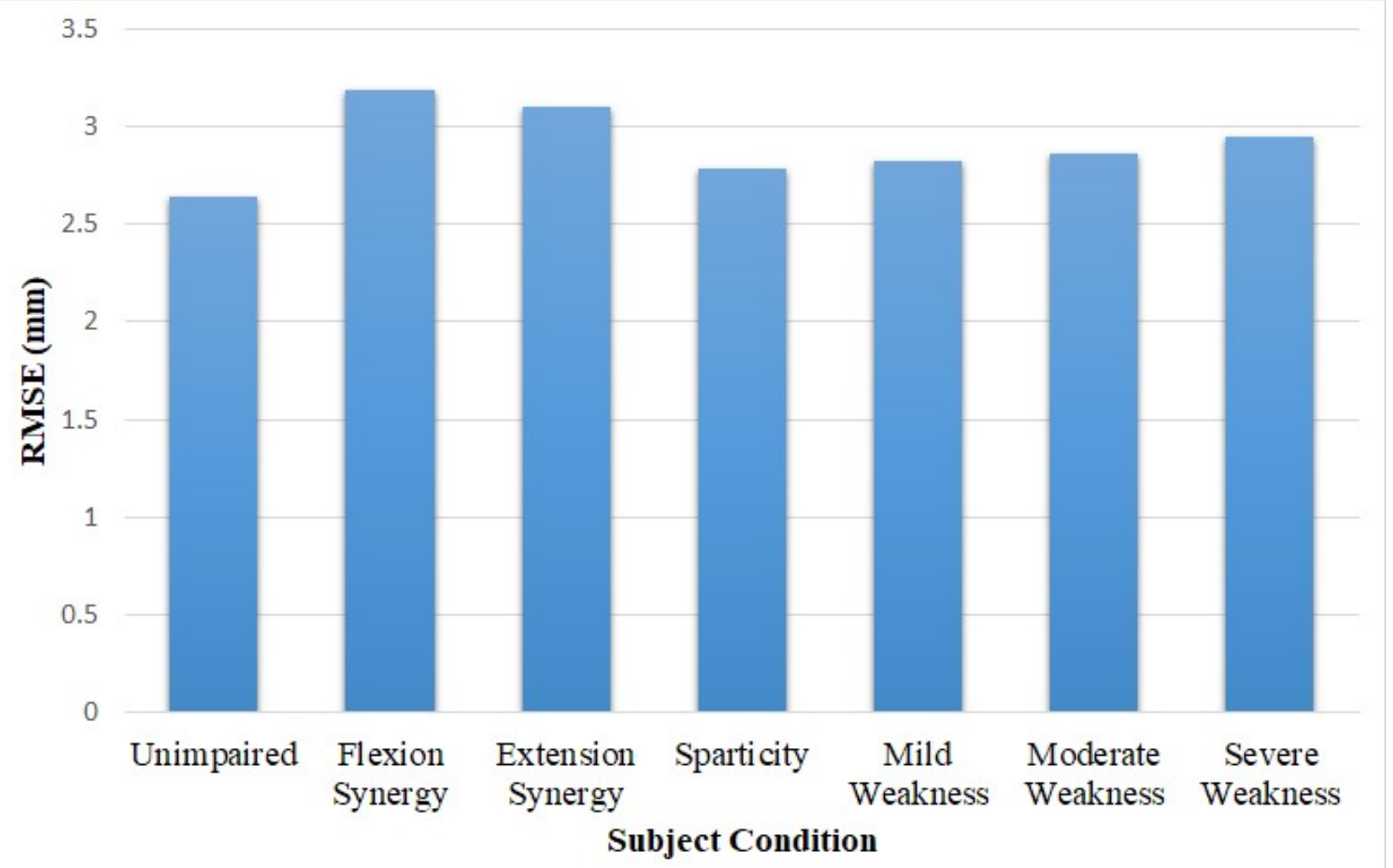

Figure 10: RMSE for the trajectories of 7 different simulated users in TC mode with a different robot dimensions.

ellipse with relatively high eccentricity (0.94) was used in order to minimise sideways movement of the arm, thereby limiting forearm rotation, which made the inverse dynamic computations more difficult to converge. The simulated normal subject was unable to trace the trajectory perfectly, which is similar to what happens in real humans. For comparison, we recruited a single subject to move an actual version of the PULSR robot, with a ball-point pen attached near the end effector to trace movement. The resulting traces are shown in Fig. 6(b).

Trajectories for various biomechanical arm models moving the robot model in FML mode are shown in Fig. 7. For the flexion synergy and extension synergies (Fig. 7(a) and (b)), the eliptical movements are flattened during the inward and outward movements of the arm respectively. This correctly reflects the fact that flexion is accompanied by shoulder abduction and forearm supination for flexion synergy, while in extension synergy, elbow extension, shoulder adduction, and forearm pronation all take place together [28]. The spasticity model experienced notably increased jerking during elbow extension, as can be seen from Fig. 7(c). The three muscle weakness models however seemed to have no discernable pattern of systematic errors. This is why all three conditions are represented by the trajectories for moderate weakness in Fig. 7(d). This may be explained by the fact that muscle weakness would normally affect the pace at which the trial is completed, rather than the actual errors.
The RMSE error for each subject during FML mode are shown in Fig. 8. Flexion and extension synergies have higher errors because of the nature of movement impairment. The jerky movement of the spasticity model during arm extension result in the the impaired trajectory error varying intermittently. Similary, for the weakness models, the errors are also more random than the synergy models. For the synergy models, there is a strong and persistent deviation during extension or flexion, which contributes to the increased error. As previously mentioned, the difference in mild, moderate and severe weakness are not pronounced. For comparison, the RMSE errors for TC mode, in which the robotic arm was actively working to assist the arm models, are presented in Fig. 9.

The errors for all subjects reduced, with movement synergy and spasticity impairments most affected. The primary take-away from this should be the fact that the interactions of the biomechanical models and robot model resulted in a reduction of the movement errors, just as a real human and real robot would. Not much can be read into the actual magnitude of the errors, as will be explained later in this section.

In order to determine whether the dimensions of the robot affects performance, the geomety of the robot model was altered, such that Link 1, Link 2 and Link 3 were $0.4 \mathrm{~m}$ long, while Link 4 remained $0.6 \mathrm{~m}$. The simulation was repeated in TC mode, and the resulting RMSE errors are presented in Fig. 10. A single factor ANOVA test 
of the RMSE errors from TC mode of the two different geometries resulted in a p-value of 0.479 , $\mathrm{F}=0.533$, F-crit $=4.74$, which means that the null hypothesis cannot be rejected that both sets of errors are from the same population. In other words, changing the geometry of the robot (by modifying its dimensions) did not significantly affect the performance of the simulation.

An important limitation of this study will now be discussed. There is at present no substantial data available on the quantitative measures of deficit in stroke impairments. For that reason, the trajectories generated and discussed in the foregoing were discussed in light of the theoretical understanding of the various conditions. In addition, this prevents a more thorough comparison of the RMSE errors as mentioned previously. Additional studies are therefore planed in which stroke patients will be recruited to validate the biomechanical models in this study.

\section{CONCLUSION}

Modeling and simulation can lead to significant reduction in development and testing costs in terms of time, money, and other resources. The introduction of modelling frameworks capable of capturing the interaction between human subjects and robots has been challenging due to the complexity of accurately modelling the guman operator. By using biomechanical models of the human arm following pre-stipulated trajectorytracing tasks, this study minimized the role of human volition and demonstrated a way in which control laws for rehabilitation robots can be tested more rapidly, by eliminating the usual system identification process needed to configure a robot to a real human user. While a lot more work needs to be done in this area, this study has demonstated the possibility and the promise of simulating human-robot interaction during hand rehabilitation.

\section{Acknowledgement}

This research was sponsored by a TETFUND National Research Fund grant. The authors thank the Sanofi Family Foundation and Prof. P. Ogunbona for the HP Z440 workstation used for the conduction of this study. The Titan Xp GPU of the workstation was donated by the Nvidia Corporation.

\section{References}

[1] H. I. Krebs, L. Dipietro, S. Levy-Tzedek, S. E. Fasoli, A. Rykman-Berland, J. Zipse, J. A. Fawcett, J. Stein, H. Poizner, and A. C. Lo, "A paradigm shift for rehabilitation robotics," IEEE engineering in medicine and biology magazine, vol. 27, no. 4, pp. 61-70, 2008.

[2] G. Kwakkel, B. J. Kollen, and J. I. Krebs, "Effects of robot-assisted therapy on upper limb recovery after stroke: a systematic review," Neurorehabilitation and neural repair, vol. 22 , no. 2, pp. 111-121, 2008.

[3] S. Masiero, M. Armani, and G. Rosati, "Upper-limb robot-assisted therapy in rehabilitation of acute stroke patients: focused review and results of new randomized controlled trial," J Rehabil Res Dev, vol. 48, no. 4, pp. 355-366, 2011.
[4] T. J. Wolf, M. Doherty, A. Boone, J. Rios, H. Polatajko, C. Baum, and S. McEwen, "Cognitive oriented strategy training augmented rehabilitation (costar) for ischemic stroke: a pilot exploratory randomized controlled study," Disability and rehabilitation, pp. 1-10, 2019.

[5] B. Sheng, W. Meng, C. Deng, and S. Xie, "Model based kinematic and dynamic simulation of 6-dof upper-limb rehabilitation robot," in 2016 Asia-Pacific Conference on Intelligent Robot Systems (ACIRS). IEEE, 2016, pp. $21-25$.

[6] L. Lia, Z. Xiang, H. Liua, Y. Shao, and J. Zhang, "Design and simulation of lower limb rehabilitation robot based on human physiological characteristics," in Transdisciplinary Engineering: A Paradigm Shift: Proceedings of the 24th ISPE Inc., vol. 5, International Conference on Transdisciplinary Engineering. IOS Press, July 2017, p. 428.

[7] P. Maciejasz, J. Eschweiler, K. Gerlach-Hahn, A. Jansen-Troy, and S. Leonhardt, "A survey on robotic devices for upper limb rehabilitation," Journal of neuroengineering and rehabilitation, vol. 11, no. 1 , pp. 3-32, 2014

[8] L. Marchal-Crespo and D. J. Reinkensmeyer, "Review of control strategies for robotic movement training after neurologic injury," Journal of neuroengineering and rehabilitation, vol. 6, no. 1, pp. 1-15, 2009.

[9] A. Duschau-Wicke, A. Caprez, and R. Riener, "Patientcooperative control increases active participation of individuals with sci during robot-aided gait training," Journal of neuroengineering and rehabilitation, vol. 7, no. 1,2010

[10] K. P. Jankowski, Inverse dynamics control in robotics applications. Trafford, 2004.

[11] M. Damsgaard, J. Rasmussen, S. T. Christensen E. Surma, and M. DeZee, "Analysis of musculoskeletal systems in the anybody modeling system," Simulation Modelling Practice and Theory, vol. 14, no. 8, pp. 11001111,2006

[12] R. Davoodi and G. E. Loeb, "Msms software for vr simulations of neural prostheses and patient training and rehabilitation," in MMVR, 2011, pp. 156-162.

[13] S. L. Delp and J. P. Loan, "A graphics-based software system to develop and analyze models of musculoskeletal structures," Computers in biology and medicine, vol. 25 , no. 1 , pp. 21-34, 1995.

[14] S. L. Delp, F. C. Anderson, A. S. Arnold, P. Loan A. Habib, C. T. John, E. Guendelman, and D. G. Thelen, "Opensim: open-source software to create and analyze dynamic simulations of movement," IEEE transactions on biomedical engineering, vol. 54 , no. $11, \mathrm{pp}$ 1940-1950, 2007.

[15] E. K. Chadwick, D. Blana, R. F. Kirsch, and A. D. van den Bogert, "Real-time simulation of threedimensional shoulder girdle and arm dynamics, IEEE Transactions on Biomedical Engineering, vol. 61, no. 7 , pp. 1947-1956, 2014.

[16] F. E. Zajac, "Muscle and tendon: properties, models, scaling, and application to biomechanics and motor control," Critical reviews in biomedical engineering, vol. 17, no. 4, pp. 359-411, 1989.

[17] J. He, W. Levine, and G. E. Loeb, "Feedback gains for correcting small perturbations to standing posture," in Proceedings of the 28th IEEE Conference on Decision and Control. IEEE, 1989, pp. 518-526.

[18] C. T. Freeman, E. Rogers, J. H. Burridge, A. Hughes, and K. L. Meadmore, Iterative learning control for electrical stimulation and stroke rehabilitation. Springer, 2015

[19] J. W. Krakauer, "Arm function after stroke: from physiology to recovery," in Seminars in neurology, vol. 25. 333 Seventh Avenue: Thieme Medical Publishers, Inc., 2005, pp. 384-395.

[20] M. Afschrift, F. DeGroote, J. De Schutter, and I. Jonkers, "The effect of muscle weakness on the capability gap during gross motor function: a simulation study supporting design criteria for exoskeletons of the lower limb," Biomedical engineering online, vol. 13, no. 1, pp. 359-411, 2014

[21] C. F. Ong, T. Geijtenbeek, J. L. Hicks, and S. L. Delp, 
"Predicting gait adaptations due to ankle plantarflexor muscle weakness and contracture using physics-based musculoskeletal simulations," PLoS computational biology, vol. 15, no. 10, p. e1006993, 2019.

[22] T. K. Koo and F. T. Mak, "A neuromusculoskeletal model to simulate the constant angular velocity elbow extension test of spasticity," Medical engineering and physics, vol. 28 , no. 1 , pp. 60-69, 2006.

[23] A. Falisse, L. Bar-On, K. Desloovere, I. Jonkers, and F. De Groote, "A spasticity model based on feedback from muscle force explains muscle activity during passive stretches and gait in children with cerebral palsy," PloS one, vol. 13, no. 12, pp. 1-20, 2018.

[24] M. J. Kang, C. S. Shin, and H. H. Yoo, "Modeling of stretch reflex activation considering muscle type," IEEE Transactions on Biomedical Engineering, vol. 65, no. 5, pp. 980-988, 2017.

[25] C. Trompetto, L. Marinelli, L. Mori, E. Pelosin, A. Currà, L. Molfetta, and G. Abbruzzese, "Pathophysiology of spasticity: implications for neurorehabilitation," BioMed research international, p. 354906, 2014.

[26] L. H. Ting and J. L. McKay, "Neuromechanics of muscle synergies for posture and movement," Current opinion in neurobiology, vol. 17, no. 6, pp. 622-628, 2007.

[27] L. Dipietro, H. I. Krebs, S. E. Fasoli, B. T. Volpe, J. Stein, C. Bever, and N. Hogan, "Changing motor synergies in chronic stroke," Journal of neurophysiology, vol. 98, no. 2, pp. 757-768, 2007.

[28] P. Kung, C. K. Lin, and M. Ju, "Changing motor synergies in chronic stroke: Neuro-rehabilitation robotassisted assessments of synergy patterns of forearm, elbow and shoulder joints in chronic stroke patients," vol. 25 , no. 7 , pp. $647-654,2010$.

[29] C. T. Freeman, A. M. Hughes, J. H. Burridge, P. Chappell, P. L. Lewin, and E. Rogers, "A robotic workstation for stroke rehabilitation of the upper extremity using fes," Medical Engineering and Physics, vol. 31, no. 3, pp. 364-373, 2009. 